\title{
Is there a Connection Between Non-Synchronous Rotation and X-Ray Emission in Massive Binary Systems?
}

\author{
Sinhué Haro ${ }^{1}$, Juan Antonio Juárez ${ }^{1}$, and Gloria Koenigsberger ${ }^{2}$ \\ 1 Instituto de Astronomía, UNAM, Mexico D.F. 04510; ${ }^{2}$ Centro de \\ Ciencias Físicas, UNAM, Cuernavaca, Morelos, 62251
}

\begin{abstract}
A correlation between orbital period and $\log \left(\mathrm{L}_{\mathrm{X}} / \mathrm{L}_{\text {bol }}\right)$ is found for a sample of B-type binary systems. We suggest that wind-wind collisions are the likely mechanism for generating the X-ray emission, and that the mass-loss rates may be enhanced in non-synchronously rotating systems due to the oscillations that are excited by the tidal forces.
\end{abstract}

Non-radial oscillations are excited in stars in binary systems where the orbital period $\left(\mathrm{P}_{\text {orb }}=2 \pi / \Omega\right)$ is not synchronized with the stellar rotational period $\left(\mathrm{P}_{\text {rot }}=2 \pi / \omega\right)$. In this paper we explore the possibility of an association of these oscillations with X-ray emission. When $\mathrm{P}_{\text {rot }}=\mathrm{P}_{\text {orb }}$, the system is in equilibrium and no oscillations are expected. A simple model for tidal interactions (Moreno \& Koenigsberger 1999; Moreno et al. 2003) predicts that, excluding resonances, the maximum amplitudes of oscillation occur when $\mathrm{P}_{\text {rot }}$ approaches the value of $\mathrm{P}_{\text {orb }}$. Defining $\beta=\omega / \Omega$, the hypothesis linking X-ray emission with nonsynchronous rotation implies that in binary systems where $\beta=1$ lower X-ray luminosities should be observed, as compared to similar systems in which $\beta$ is close to, but different from unity.

In the general case of an eccentric orbit at periastron, we can write $\beta=$ $0.02 \frac{v_{\mathrm{e}} /(\mathrm{km} / \mathrm{s})}{R_{*} / R_{\odot}}\left(P_{\mathrm{orb}} /\right.$ days $) \frac{(1-e)^{3 / 2}}{(1+e)^{1 / 2}}$, where $v_{\mathrm{e}}$ is the equatorial velocity of the star, $\mathrm{R}_{*}$ its radius, and $\mathrm{e}$ is the eccentricity of the orbit. There are two major sources of uncertainty in $\beta$. The first is that the observational data generally provide only $v \sin i$. But, many of the binary systems are eclipsing, which provides a strong lower limit to $v \sin i$. The second problem concerns the radius of the star, although this problem is less severe if we concentrate on B-type stars, as opposed to O-type stars. In Figure 1 we plot the values of $\log \left(\mathrm{L}_{\mathrm{X}} / \mathrm{L}_{\mathrm{bol}}\right)$ vs. $\beta$ for B-type binaries in circular orbits having values of $v \sin i$ measured by Abt et al. (2002) and which were also detected by ROSAT (Berghoefer et al. 1996). Of the $\sim 220$ binaries listed by Abt et al. (2002), $\sim 26$ were detected by ROSAT. This sample is reduced to 8 objects when we restrict it to binaries with luminosity classes IV and V and with circular $(e<0.11)$ orbits. Fig. 1 displays a difference of two orders of magnitude in $\log \left(\mathrm{L}_{\mathrm{X}} / \mathrm{L}_{\mathrm{bol}}\right)$ for the different systems, with a trend for lower $\log \left(\mathrm{L}_{\mathrm{X}} / \mathrm{L}_{\mathrm{bol}}\right)$ associated with two of the three systems that have $\beta$ closest to unity. This trend is not unique, however, since there is also a trend with orbital period, as illustrated in the top right panel of Fig. 1. The bottom right panel illustrates the behavior of our sample as a function of $v \sin i$, where a weak trend for increasing $\log \left(\mathrm{L}_{\mathrm{X}} / \mathrm{L}_{\mathrm{bol}}\right)$ with $v \sin i$ might be present. This trend is certainly not present in the B8-9 single stars in Abt et al.'s list that were 
detected by ROSAT (plotted as open squares in Fig. 2). Hence, we tentatively conclude that there is a correlation between $\log \left(\mathrm{L}_{\mathrm{X}} / \mathrm{L}_{\mathrm{bel}}\right)$ and orbital period, for $2<P_{\text {orb }}<8$ and that a possible trend for lower X-ray emission in $\beta=1$ systems may be present.

Finally, there remains the issue of the mechanism responsible for generating the X-rays. In massive binary systems in which wind-wind collisions occur, the emitted X-ray luminosity is predicted to increase as the orbital separation decreases. The correlation of $\log \left(\mathrm{L}_{\mathrm{X}} / \mathrm{L}_{\text {bol }}\right)$ vs. $\mathrm{P}_{\text {orb }}$ is consistent with this prediction, and hence suggests that wind-wind collisions may be responsible for the observed X-ray emission. In addition, an indirect correlation between the $\mathrm{X}$-ray emission and $\beta$ may exist since large amplitude stellar pulsations could contribute towards enhancing the mass-loss rate of the stars, thus producing a more powerful collision between the two winds than in the case of a synchronized binary system.

Acknowledgements. Participation in this Symposium was made possible by the Posgrado en Astronomía (UNAM), CLAF, CCF-UNAM, and CONACYT grant 36359 , to all of which we express our appreciation.

\section{References}

Abt, Levato, Grosso 2002, ApJ 573, 359

Batten, A.H., Fletcher, J.M., \& MacCarthy, D.G. 1989, Eighth Catalogue of the Orbital Elements of Spectroscopic Binary Systems (Publ. Dom Astrophys. Obs. Victoria 17; Victoria:DOA)

Berghoefer, T.W., Schmitt, J.H.M.M., Cassinelli, J.P. 1996, A\&AS 118, 481.

Moreno, E. \& Koenigsberger, G. 1999, RMA\&A 35, 157

Koenigsberger, G., Moreno, E., \& Cervantes, F., 2002, in IAU Symposium \# 212, van der Hucht, Herrero \& Esteban (ed.), p. 101

Table 1. B-star binaries with nearly circular orbits and ROSAT detections

\begin{tabular}{rrllllllll}
\hline HR & HD & Sp. & $\frac{R}{R_{\odot}}$ & vsini & $\log \left(\mathrm{L}_{\mathrm{X}}\right)$ & $\log \left(\frac{L_{\mathrm{X}}}{L_{\mathrm{bol}}}\right)$ & $\mathrm{P}_{\text {orb }}$ & $\mathrm{e}$ & $\beta$ \\
\hline 936 & 19356 & B8V & 2.1 & 50 & 30.82 & -5.13 & 2.87 & 0.02 & 1.37 \\
1339 & 27295 & B9IV & 2.0 & 20 & 30.05 & -5.73 & 4.45 & 0.06 & 0.89 \\
1347 & 27376 & B9V & 2.0 & 20 & 28.54 & -7.00 & 5.01 & 0.01 & 1.00 \\
1471 & 29365 & B8V & 2.1 & 70 & 31.41 & -4.46 & 2.05 & 0.0 & 1.37 \\
1657 & 32964 & B9V & 2.0 & 30 & 29.88 & -5.71 & 5.52 & 0.10 & 1.66 \\
1788 & 35411 & B1V & 6.5 & 35 & 31.57 & -6.93 & 7.98 & 0.0 & 0.86 \\
7326 & 181182 & B8III & 8.0 & 60 & 31.56 & -4.72 & 3.38 & 0.03 & 0.51 \\
7792 & 193964 & B9V & 2.0 & 125 & 30.17 & -5.42 & 5.30 & 0.04 & 6.62 \\
\hline
\end{tabular}



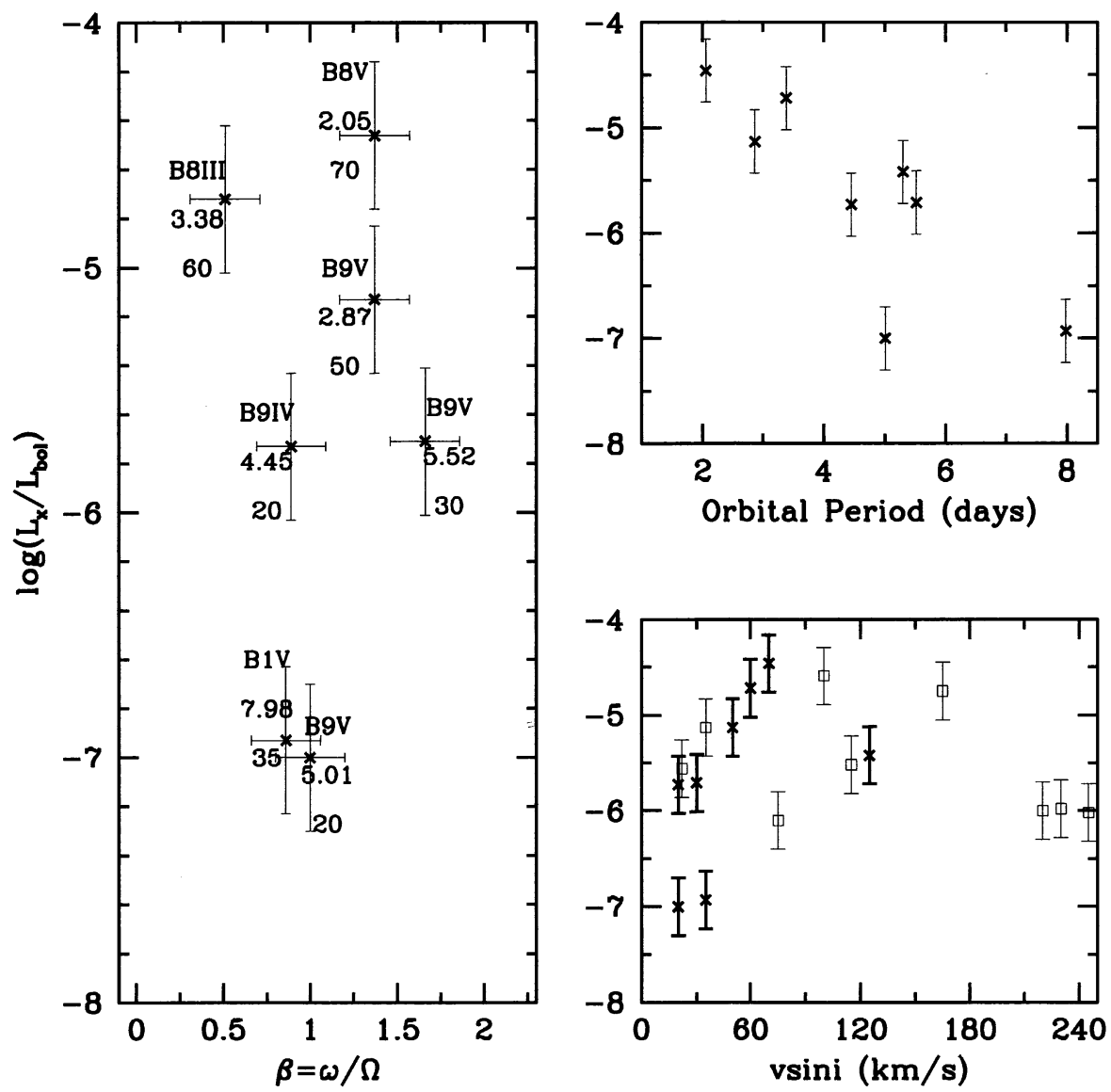

Figure 1. $\quad \log \left(\mathrm{L}_{\mathrm{X}} / \mathrm{L}_{\mathrm{bol}}\right)$ as a function of $\beta$, orbital period and $v \sin i$ for a sample of B-star binaries (crosses) from the catalogue of Abt et al. (2002). The open squares are single B8-9V stars with X-ray detections in Berghoefer et al. (1996). The decreasing trend with orbital period is reminiscent of the effects expected due to wind-wind collisions. 